Dupuis, Gilles, "Pour une herméneutique du style », dans Jean-François Vallée, Jean Klucinskas et Gilles Dupuis (dir.), Transmédiations. Traversées culturelles de la modernité tardive, Montréal, Les Presses de l’Université de Montréal, 2012, p. 192-213. 


\title{
Pour une herméneutique du style
}

\author{
Gilles Dupuis
}

\begin{abstract}
T U COMMENCEMEnT ÉTAIT LE STYLe, et le style était monolithique, et Ale monolithe était style. Mais au commencement de quels commencements? L'Odyssée immémoriale d'Homère ou celle, postmémorielle, de Stanley Kubrick? L'odyssée qui nous fait traverser les eaux troubles du Léthé ou celle qui nous plonge dans la source solennelle du Styx? Le style descendant sur terre sous la forme d'un monolithe pour accélérer le développement de la parole chez les hommes singes ou celui, létal, remontant le fleuve héraclitéen afin de la ravir, tel le feu prométhéen, aux sages femmes? Premier postulat axiologique: la question du style est elle-même une question de style ${ }^{1}$. Impossible de la soulever sans d'abord reconnaître qu'elle dépend de sa formulation dans l'avancement hasardeux d'une réponse. C'est pourtant ce à quoi je vais m'employer, avec ou sans style, dans les pages qui suivent. À défaut de répondre à la question «qu'est-ce que le style?» ou "qu'en est-il du style aujourd'hui?», je veux interroger le style comme opération herméneutique manœuvrant entre forme et $\mathrm{sens}^{2}$. La question ainsi reformulée devient: que peut faire le style?

Que fait donc le style? Avançons une première réponse péremptoire: la différence. Il faut revenir au lieu commun en forçant un peu l'écoute si nous voulons entendre son effet de vérité. Le style fait la différence. Cela ne veut pas dire que, sans style, il n'existe pas de différence; cela revient plutôt à dire que, dès que point le style, apparaît nécessairement la différence. Pas seulement une dissemblance, comme il y a de ces différences
\end{abstract}

1. Il s'agit moins ici de poser la «question du style», comme le faisait Derrida dans Éperons. Les styles de Nietzsche (Paris, Flammarion, coll. «Champs», 1978), que de faire de cette question une affaire de style.

2. Cette hypothèse a déjà été explorée par Ricœur dans ses travaux portant sur le texte, où il prône la conciliation de l'approche herméneutique avec la démarche structuraliste. Voir Paul Ricœur, "Qu'est-ce qu'un texte?», Du texte à l'action. Essais d'herméneutique II, Paris, Seuil, coll. «Esprit», 1986, p. 137-159. 
discrètes, voire notoires, d'un sujet à un autre, mais l'altérité irréductible en tant que singularité à l'œuvre. Si tout le monde a un style, une façon d'être particulière, une personnalité, un tempérament ou un caractère distinct, ce n'est pas tout ce beau monde, loin s'en faut, qui arrive à signer cette soi-disant individualité. L'expression même "tout le monde», pour signifier chacun chacune qui le compose, indique à quel point il est difficile de discerner le spécimen de l'espèce, l'individu de la masse. Encore faut-il faire la distinction entre style et «style», ou style et contrefaçon: d'une part, la stylisation, à savoir l'esthétisation du style; de l'autre, la signature, soit sa manifestation singulière. Le style advient quand, paradoxalement, l'individu se déprend de son individualité factice, quand il cesse de se fantasmer unique pour le devenir réellement. Le style survient quand l'individu renonce à se concevoir comme indivis et acquiesce à la division du sujet qui en fait, en vérité, un dividu ${ }^{3}$. Alors, seulement, le style peut-il surgir, même si rien ne garantit $a$ priori le succès de l'opération.

Mais laissons là les considérations intempestives qui concernent l'absoluité du phénomène. Revenons plus concrètement à notre problème de départ en l'infléchissant quelque peu: que peut faire le style quand il ne se contente pas de servir une mode, une école ou un mouvement, lorsqu'il ne se cantonne plus dans sa fonction ornementale; bref, quand il ne se laisse pas réduire à un simple accessoire ni résumer à une nouvelle tendance? Le style, à lui seul, indépendamment de ses autres fonctions, peut réaliser une trouvaille susceptible de lui conférer une valeur heuristique. C'est le critère de performativité qui entre ici en jeu. Une toile de Rothko, une pièce de Ligeti ou une page de Quignard frappe d'abord, avant toute interprétation de l'œuvre, comme une pétition de principe en faveur du style, puisque ce qu'il s'agit de démontrer ne peut en fait qu'être montré. L'indice du style, c'est un index visible, lisible ou audible qui pointe pour attirer l'attention dans la direction où ça se passe. Une signature s'affiche, résonne, s'étale; moins qu'incomparable, elle est irréfutable. Appelons cela du style incarné ou plutôt de l'art incarné par le style. À l'autre bout du spectre, il y a le style désincarné, celui qui fait journal, menu, carte de mode, modèle académique. Le style passe-partout, prêt-à-porter tout aussi prêt à jeter, l'industrie produisant du style comme n'importe quelle autre matière à consommer, éventuellement à recycler, comme cela se fait de plus en plus à l'université. Toujours pour dire qu'il y a style... et style. Il faut entendre la différence dans l'apparente redondance. Mais je glisse de nouveau sur la pente de

3. Selon les sources consultées en ligne, le néologisme est tantôt attribué à Gilles Deleuze, tantôt dérivé d'un jeu de mots lacanien sur in-dividu («Un-dividu»). Peu importe l'origine de la «trouvaille», c'est sa valeur heuristique qu'il convient ici de souligner. Un pas de plus et on établirait une équivalence entre style et schize du sujet. 
l'absolu où je risque de tourner en rond, pris vicieusement dans un cercle faussement herméneutique.

Nouvelle tentative de remettre cette réflexion sur ses rails: si le style peut faire une différence, en quoi consiste $s a$ trouvaille? La possibilité heuristique du style, qui nous pousse à crier: "Eurêka!» chaque fois qu'elle se réalise, est précisément ce qui nous permet d'approcher son mystère d'un point de vue herméneutique - et non seulement stylistique. Si le style peut faire une différence au point d'engendrer de l'inédit, de l'inouï, du jamais $\mathrm{vu}$ - ce qui n'est tout de même pas la même chose que célébrer le génie ou vouer un culte à l'originalité -, c'est qu'il est aussi producteur, porteur ou à tout le moins vecteur de sens. Le sens, bien entendu, n'est pas uniquement un produit de la pensée que l'on recherche ensuite dans le «contenu» des œuvres où il est censé avoir été déposé par cette dernière ou du côté des intentions de l'auteur qui aurait voulu communiquer à son lecteur le fruit de ses pensées (ses supposées idées «originales $\left.{ }^{4} »\right)$; il ne se ramène pas plus au seul «contenant» que constituerait la mise en forme de l'Idée ayant présidé à la réalisation de l'CEuvre, selon une conception platonicienne ou métaphysique du monde. Le sens est tout à la fois cela, mais plus encore. Il est le résidu qui reste à interpréter quand on a tout épluché de l'œuvre et de l'auteur, après avoir compulsé les annales de l'Histoire. Ce reste, qui a échappé à notre attention, pourrait se trouver là où personne n'a songé à le chercher: à même les mailles du style - à condition que ce dernier soit bien davantage que le bel enrobage dans lequel l'œuvre nous a été livrée.

Nous y sommes (enfin presque): le style comme créateur de sens «ex nihilo ${ }^{5}$. À même la vacuité du dire proféré par la bêtise des "parlêtres» (Lacan), à même le babil recyclé des «écrivants» (Barthes) - et j'ajouterais nombre d'écrivains qui se prennent pour des auteurs -, se dit quelque chose qui, en vertu de son agencement, sonne à la fois juste et neuf. Il ne s'agit pas d'une formule incantatoire (bien que cela puisse en prendre la forme), ni d'une formalisation arithmétique (ici je prends congé de Lacan et de ses mathèmes), mais d'un composé qui ne semble pas avoir existé avant sa manifestation singulière. Je dis bien: "ne semble pas avoir existé». Mais comment le savoir? Pas moyen de le savoir, justement, aurait avancé Bataille en se réclamant de la docte ignorance d'un Nicolas de Cues. Pas moyen de le vérifier, peut-être, mais possibilité de le reconnaître. C’est ici

4. Rien de plus vulgaire que les idées, prétendait Céline. Tout le monde en a, mais très peu ont du style. Or, lui, il était «un homme à style». Voir Louis-Ferdinand Céline, «Ma grande attaque contre le Verbe», Le style contre les idées, Bruxelles, Éditions Complexe, coll. «Le Regard littéraire», 1987, p. 61-73.

5. Au sens où Lacan entend l'expression quand il glose la célèbre formule de Picasso: «Je ne cherche pas, je trouve.» Voir Jacques Lacan, «De la création ex nihilo», Le Séminaire, livre VII. L'éthique de la psychanalyse, Paris, Seuil, coll. «Le champ freudien», 1986, p. 39-152. 
que le jugement doit intervenir, en s'éclairant de la plus vaste culture qu'il nous soit loisible d'assimiler en traversant toutes les époques accessibles à notre mémoire collective (aussi défaillante soit-elle). On reconnaît le style à d'infimes nuances par rapport à du déjà donné, bien plus qu'à une mise en scène criarde effectuée par un artiste pompier (encore que ce dernier puisse, à l'occasion, faire mouche sans renoncer au kitsch...). Ne pourraiton pas concevoir le style, si l'on tient à le maintenir en orbite transcendantale, comme la différence ontologique d'un Heidegger qui, dans sa formulation avancée par Vattimo, signifie la persistance du même (das Selbe), mais en tant qu'il n'est pas égal (das Gleiche) à lui-même ${ }^{6}$ ? Certes, mais comme il s'agit de mesurer - «jauger» serait plus juste que «juger» - une différence à l'œuvre, à la lettre une manœuvre relevant du réel (le domaine de l'ontique), je préfère prendre le phénomène, voire l'épiphénomène, par l'autre bout de la lorgnette, en insistant sur le degré de variation qu'il implique dans sa manifestation. Dût-elle se réduire à un écart infinitésimal, la variation n'en resterait pas moins tangible: en elle réside la part de réel du style.

Chaque nouvelle spire de cette spirale par laquelle je tente de nous rapprocher de l'objet sondé, tout en m'en éloignant temporairement, nous ramène au plus près de sa question. Mais je sens que si je ne passe pas bientôt à des exemples «concrets», je vais perdre mon lecteur... et moi de même. Nouvelle tentative donc - et à ce rythme, vous aurez compris que ce jeu pourrait se poursuivre à l'infini - de revenir au nerf de la chose plus qu'au nœud de la question (le nerf étant d'autant plus réceptif qu'il se trouve en surface du corps) pour montrer en quoi le style peut encore faire une différence. Je prendrai mes exemples dans deux sphères distinctes de l'art qui, à bien des égards, semblent à l'antipode l'une de l'autre, bien qu'elles se soient souvent intersectées au cours des siècles: la littérature - je précise que je donne à ce terme toute l'extension qu'il peut recevoir quand on le considère comme un art du langage - et la musique, que je limiterai toutefois pour les besoins de la démonstration à celle qui se passe de mots, soit la musique instrumentale (entendu que la voix, sans tenir compte du texte, reste un instrument).

6. «[...] autant dire, de façon plus simple, qu'il n'y a diversité dans l'histoire que s'il y a un Même qui ne se laisse pas réduire à l'Égal, c'est-à-dire qui demeure non-dit et non-pensé, et cependant toujours Même.» Gianni Vattimo, Les aventures de la différence, trad. de l'italien par Pascal Gabellone, Riccardo Pineri et Jacques Rolland, Paris, Minuit, coll. «Critique», 1985 , p. 183 


\section{L’«impure» littérature versus la musique "pure»}

Si j'insiste pour m'en tenir à ces deux manifestations artistiques comme modèles opératoires, c'est qu'en plus de constituer un réservoir inépuisable d'exemples stylistiques à travers les âges, elles sont diamétralement opposées en ce qui concerne, de prime abord, la question de l'herméneutique. Si la littérature peut difficilement se passer du sens, et donc de l'interprétation - les œuvres littéraires les plus absurdes, incohérentes ou qui cultivent à l'extrême l'«insignifiance» s'inscrivent tout de même dans la tradition du sens, ne serait-ce qu'en faisant le procès de cette tradition -, en revanche, la musique, lorsqu'elle se prive du texte, arrive difficilement à signifier autre chose qu'elle-même. Le terme même d'interprétation prend un sens différent selon que le contexte est littéraire ou musical. Qu'il soit conçu comme intention de l'auteur, effet de l'œuvre ou perception du lecteur, le sens littéraire est toujours de l'ordre d'une signification à découvrir (déchiffrer, analyser, expliquer, exposer, etc.). Qu'on l'appréhende par le fond ou par la forme, on ne peut faire l'économie du langage qui constitue son matériau de base. Or, comme les mots signifient, quel que soit l'usage qu'on leur destine, ils ne peuvent échapper complètement au travail herméneutique. Interpréter, en littérature, consiste toujours à s'interroger sur le sens d'une page, d'une phrase, d'un vers, même (surtout) quand les questions restent sans réponses. En musique, interpréter revient à jouer une pièce quelconque, à exécuter le morceau choisi. L'interprète n'est pas le musicologue qui se penche éventuellement sur le sens probable ou possible d'une partition, mais le musicien qui « réalise» cette partition lors d'une performance. En principe, cette opération échappe à l'herméneutique, à moins de la concevoir (par exemple chez Glenn Gould) comme une analyse en acte de l'œuvre musicale, réponse performative à une question rhétorique non formulée. Mais, réduite à sa plus simple expression - des sons et des silences transcrits par des notes et des soupirs -, la musique reste asémantique. Le compositeur, comme par ailleurs l'interprète, le critique ou l'auditeur, peut vouloir insuffler du sens dans cette matière sonore, il n'en reste pas moins que cette dernière en est privée en soi et ne peut y prétendre que par métaphore ${ }^{7}$.

Bien sûr, la littérature ne se laisse pas réduire à sa fonction sémantique; la musique, bien entendu, n'est pas absolument réfractaire au sens. Sans tenir compte des œuvres musicales qui font appel au texte, partant à la signification (motets, cantates, messes, chansons, madrigaux, opéras, ora-

7. Voir à ce sujet l'introduction de Jean-Jacques Nattiez à son ouvrage: La musique, les images et les mots. Du bon et du moins bon usage des métaphores dans l'esthétique comparée, Montréal, Fides, coll. «Métissages», 2010, p. 9-30. 
torios, etc.), il existe en Occident toute une tradition que l'on désigne vaguement par l'expression «musique à programme». Descriptive ou simplement suggestive, évocatrice ou invocatrice, elle a donné naissance, entre autres, à des concertos célèbres de Vivaldi, aux sonates et aux symphonies sous-titrées de Beethoven, au genre romantique du "poème symphonique» (que l'on traduit aussi en anglais, en faisant appel à une autre forme artistique, par Tone Poem ou Tone Picture), aux ballets et musiques de scène, ainsi qu'à toute une pléthore de pièces instrumentales dites d'ambiance ou de caractère. Si bien que ce qui tente le musicien semble plus souvent le moyen de faire sens avec un matériau a priori asémantique plutôt que se livrer à son exploitation sans alibi littéraire en composant de la «musique pure», alors que l'écrivain doit faire violence à la langue (et possiblement à lui-même) pour qu'elle cesse de signifier comme moyen de communication ou d'expression et s'ouvre au non-sens délivré de la tentation exégétique.

$\mathrm{Si}$, donc, la musique recherche ce dont elle est privée par définition et que la littérature échappe difficilement à ce qui la constitue par convention, qu'en est-il du style que ces deux modes d'expression artistique cultivent avec plus ou moins de bonheur? Que ce soit au sens large du terme (une techné) ou au sens restreint (l'antique poiesis), l'art n'est pas nécessairement remarquable du point de vue stylistique et n'a pas d'ailleurs à l'être. Une œuvre d'art peut nous intéresser pour de toutes autres raisons, allant du plus noble mobile au motif le plus futile. Mais quand l'œuvre capte notre attention en vertu de son style, alors il est légitime de s'interroger sur ce qui lui confère cette valeur. Qu'est-ce qui la distingue des autres œuvres d'art qui s'apparentent à elles dans le temps ou dans l'espace? En quoi un même style au sens historique du terme (baroque, classique, romantique, surréaliste, etc.) se décline-t-il différemment selon les auteurs? Quelle différence établir entre style et ton, tic et tournure, ou encore quelle pertinence accorder au style dans l'appréciation d'une œuvre? Toute une série de questions se posent à partir du postulat du style, qui ne se laissent pas facilement cerner par les réponses qu'on veut bien leur apporter.

Mais il y a une autre question, autour de laquelle je ne cesse de graviter depuis l'amorce de cette réflexion, qui surgit rarement quand on aborde le style et sur laquelle il y a lieu aussi de s'interroger: en quoi le style peutil contribuer au sens de l'œuvre; quelle est sa portée heuristique, critique, son degré théorique d'innovation? Ici, l'esthétique rencontre l'herméneutique dans un procès stylistique du sens que signale le processus signifiant du style. 


\section{L'euphonie et l'eusémie}

L'euphonie est un terme qui s'applique aussi bien à la musique qu'à la littérature. En musique, elle signifie une «harmonie de sons agréablement combinés» et s'oppose communément à cacophonie et à dissonance. Définition réductrice, car en Occident la dissonance fait partie de l'harmonie musicale dont elle constitue l'ingrédient de base avec la consonance. C'est de la combinaison judicieuse des deux que naît l'harmonie, la dissonance pratiquée pour elle-même pouvant devenir un trait stylistique chez certains compositeurs tels Gesualdo, à la Renaissance, et presque tous les compositeurs romantiques et postromantiques (du moins jusqu'à l'avènement du dodécaphonisme et l'abandon de la tonalité). En linguistique, l'euphonie désigne une «harmonie de sons qui se succèdent dans le mot ou la phrase». Pour éviter l'hiatus, le français, par exemple, introduit des sons "parasites" dont la seule fonction, asémantique, est de rendre la sonorité plus agréable ou tout simplement prononçable (tel le $t$ dans "a-t-il»). Généralisé à la littérature, et pratiqué en particulier dans la poésie, le procédé a donné lieu au jeu des allitérations et des assonances qui, avec la rime et l'anaphore, concourent à la musicalité du vers. Pour le dire en une formule lapidaire, forcément réductrice mais éloquente, l'euphonie - incluant la dissonance -, c'est l'harmonie en musique et la musique en littérature (encore faudrait-il ajouter à cette équation l'élément plus fondamental de l'eurythmie, dans tous les sens du mot).

À l'art de ce qui «sonne bien», on pourrait ajouter celui de ce qui «sonne juste» ou «signifie bien». Sur le modèle d'euphonie, je propose de forger le néologisme eusémie. L'eusémie n'est pas la leucémie du sens - sa prolifération maladive pouvant mener à la perte de tout sens ou l'asémie -, mais au contraire son économie stylistique. Ce n'est pas tant «ce qui s'énonce clairement» (pour le dire avec Boileau) que ce qui fait sens heureusement, ce qui signifie avec bonheur, ce dernier dût-il s'exprimer dans le malheur'. C'est la jouissance de la fameuse trouvaille, qui consiste en un trait d'esprit, un witz, une saillie - l'étincelle de l'intellect qui s'enflamme pour se déposer dans une forme. Une herméneutique du style, en musique comme en littérature, devient possible quand l'euphonie fait place à l'eusémie ou qu'elle l'accueille en son sein, quand l'acoustique se conjugue à la sémantique pour faire sens autrement. C'est à des signes subtils plus souvent qu'en

8. Je fais référence ici à un texte de jeunesse d'Hubert Aquin où, dans un renversement de perspectives préludant aux oxymores dont il se révélera par la suite friand, l'auteur en herbe appelait de tous ses vœux «les écrivains et les artistes capables d'aller jusqu'au bout de leur malheur d'expression!». Hubert Aquin, «Le bonheur d'expression», Mélanges littéraires II. Comprendre dangereusement, édition critique établie par Jacinthe Martel avec la collaboration de Claude Lamy, Montréal, Bibliothèque québécoise, 1995, p. 37. 
raison d'effets spectaculaires, à la lumière de lueurs incertaines plutôt qu'illuminé par des feux d'artifice, que cet «autrement» se laisse percevoir. La sensation esthétique, qui tombe sous la coupe des cinq sens (dans le cas qui nous intéresse plus précisément ici: la vue et l'ouie) et qui se prolonge en esprit, peut constituer l'indice qu'une opération herméneutique est en cours - et que le style en est le témoin privilégié, le passeur de marque. Ici, plus qu'ailleurs, le « discernement ${ }^{9} »$ est de mise. Passons donc à quelques exemples pour donner un avant-goût de ce qui pourrait constituer une analyse herméneutique du style.

Le célèbre compositeur autrichien Alban Berg, issu de la seconde école de Vienne qui donna naissance à la musique atonale, au dodécaphonisme et à la technique sérielle, s'est intéressé à la question de la compréhension musicale. Dans un article important datant de 1924, il s'interroge sur la difficulté non seulement d'écouter - et donc de recevoir - la musique de Schoenberg, mais encore d'en saisir le «sens», question que ses compositions exigeantes posent selon lui à l'entendement:

Pour répondre à cette question, l'on pourrait être tenté de rechercher les intentions mises par Schœnberg [sic] dans ses œuvres, d'examiner sa musique d'un point de vue intellectuel, de l'expliquer, comme on l'a fait trop souvent, au moyen de considérations philosophiques, littéraires ou autres. Tel n'est point mon propos. Je ne veux envisager dans les œuvres de Schœnberg que les événements proprement musicaux et le mode d'expression strictement compositionnel. Celui-ci, je l'affirme, est seul adéquat à l'objet que l'auteur a voulu figurer. N'en va-t-il pas de même pour le langage de toute œuvre d'art? C'est un postulat qu'il faut admettre au préalable: pour quiconque possède le don de penser musicalement, comprendre le langage jusqu'en ses moindres détails équivaut à comprendre l'œuvre elle-même. Grosso modo, il s'agit de reconnaître le début, le déroulement et la fin de toutes les mélodies qui la composent, d'entendre la résultante de leur simultanéité, non comme une manifestation du hasard, mais comme un ensemble d'harmonies et d'enchaînements harmoniques, d'éprouver le sens de tous les contrastes, petits et grands, en un mot, de suivre le développement du morceau ainsi qu'on suit celui d'un poème dont on possède la langue. La question formulée en tête de cet article semble donc résolue dès l'instant où l'examen du mode d'expression de Schœnberg peut nous conduire à déterminer son degré de compréhensibilitét ${ }^{10}$.

9. J'emprunte le terme à l'essai critique de Suzanne Jacob, La Bulle d'encre, Montréal, Boréal, 2001.

10. Alban Berg, Écrits, introduction, présentation et notes de Dominique Jameux, trad. par Henri Pousseur, Gisela Tillier et Dennis Collins, Paris, Christian Bourgois, coll. «musique/passé/présent», 1985, p. 24-25. Incidemment, on reconnaît dans ce passage, structuraliste avant la lettre, ce qui a permis aux théoriciens formalistes provenant de diverses disciplines de mettre sur le même plan leurs objets d'analyse pourtant très différents: à l'instar de l'inconscient lacanien, tous les arts seraient structurés comme un «langage». Il y aurait ainsi un langage musical, un langage pictural; un langage scénique, un autre cinématographique; sans oublier, bien sûr, le langage littéraire. Si elle a le mérite d'avoir permis l'avancement des 
Il faut noter, à la suite de Berg, que si le structuralisme est inévitable en musique, c'est que celle-ci est par définition un processus structurel qui se dérobe $a$ priori à la signification (au sens sémantique et non sémiotique du terme), d'où la volonté du critique de n'interroger que «les événements proprement musicaux et le mode d'expression strictement compositionnel» des œuvres. Et pourtant, Berg ouvre la voie à une herméneutique de la musique, non tant en postulant l'analogie traditionnelle entre poésie et musique via le langage (ce que les compositeurs romantiques allemands, et en particulier Wagner, avaient fait avant lui), mais en questionnant le sens même des procédés compositionnels. Il s'agit pour Berg de "penser musicalement» (que ce «don» soit inné ou acquis) et non seulement d'écouter la musique - la sentir ou la ressentir - selon les paramètres d'une expérience esthétique (ou simplement esthésique); "d'éprouver le sens de tous les contrastes " au fil de l'analyse de l'œuvre afin de «déterminer son degré de compréhensibilité». Si ce sens n'est pas donné par une sémantique, alors il faut nécessairement le débusquer dans la forme ou la structure musicale, soit un ensemble de signes et de relations établies entre eux. C'est ce que Berg s'emploie à faire dans la suite de son article en analysant en détail les dix premières mesures du Premier quatuor en ré mineur de Schoenberg, une œuvre qui appartient encore au régime de la tonalité quoiqu'il s'agisse de la tonalité élargie cultivée par le postromantisme germanique. Exemples à l'appui, le disciple montre que la difficulté, somme toute relative, de cette pièce du maître à l'époque de sa composition (1905) tient à l'asymétrie de sa structure thématique, la polyphonie des voix qui fragmente la mélodie, la grande diversité du rythme qui peut conférer à la première audition une impression d'arythmie, la richesse harmonique (une cinquantaine d'accords en une dizaine de mesures) qui entraîne une surabondance d'enchấnements et la permutation des motifs entre les voix. Conclusion: la musique tonale de Schoenberg était moins abstruse que déroutante, en bousculant les habitudes auditives du mélomane non préparé à l'accueillir ou qui ne voulait pas faire l'effort de dresser l'oreille.

Rien n'est dit explicitement du style de Schoenberg dans ce texte, style qui variera d'ailleurs en fonction des différentes périodes du compositeur qui correspondent moins au développement d'une personnalité (selon le modèle organique hérité des romantiques) qu’aux phases expérimentales

recherches formelles dans le cadre du structuralisme et de la sémiotique, cette projection d'une conceptualité héritée de la linguistique sur les autres arts n'en demeurait pas moins problématique, en particulier pour les formes artistiques qui se passent de la langue comme moyen d'expression: danse, musique, peinture, architecture... De tous les arts, seule la littérature ne peut se passer de la langue, et donc du langage au sens linguistique du mot, le théâtre pouvant quant à lui se limiter au mimodrame et le cinéma se faire muet. 
dans l'exploration de nouveaux modes de composer (de combiner les sons) et surtout de concevoir la musique en Occident. Pour saisir en quoi une herméneutique du style est possible selon Berg, il faut se tourner vers un autre texte célèbre de l'auteur de Wozzeck et Lulu: «L'impuissance musicale de la "nouvelle esthétique" de Hans Pfitzner» (1920). Dans ce texte polémique qui se veut, bien avant la réponse de Roland Barthes à Raymond Picard ${ }^{11}$, une réplique cinglante servie à cet ennemi de la "nouvelle musique»- musicien médiocre mais apprécié en son temps, auteur de Die neue Ästhetik der musikalischen Impotenz - Ein Verwesungssymptom? ( $\mathrm{La}$ Nouvelle Esthétique de l'impuissance musicale: un symptôme de décomposition?) -, Berg reprend l'exemple analysé par son «homologue » (il n'aurait sans doute pas apprécié le terme, mais «collègue» ne convient guère davantage) pour défendre et illustrer la vraie musique, soit "eine Traümerei» («Rêverie») de Schumann, pièce célébrissime tirée de son recueil Kinderszenen (Scènes d'Enfants). Tout l'argument de Pfitzner tourne autour de la «beauté» de la mélodie de Schumann et de l'impossibilité d'en rendre compte... musicalement:

Mais en présence d'une mélodie comme celle-ci, on perd complètement pied. L'on peut reconnaître sa valeur, on ne saurait la démontrer. Pour faire à son sujet l'accord des opinions, l'intelligence ne sera d'aucun secours; on est sensible à son charme ou on ne l'est pas. Nul argument n'est capable de convertir celui qui refuse d'être ravi par elle, et il n'y a rien à dire à quiconque l'attaque, sinon la lui jouer et s'écrier: «Comme c'est beau!» Ce qu'elle exprime est d'une évidence aussi profonde, d'une clarté aussi mystique que la vérité elle-même ${ }^{12}$.

Berg, qui est également sensible au «charme» de cette mélodie, ne restera pas pour autant bouche bée devant le mystère de sa beauté, ni ne perdra pied lorsqu'il s'agira d'en démontrer la valeur pour l'appréciation du morceau de Schumann. Comme pour le premier quatuor de Schoenberg, il s'attelle à l'analyse des subtilités structurelles, harmoniques, rythmiques et polyphoniques de cette courte pièce qui tient en une page afin d'en révéler toute la richesse compositionnelle que Pfitzner réduit à un maigre squelette. De tous ces procédés d'écriture, il y en a un qui ne peut s'expliquer par la structure seule de la pièce. Il s'agit de la note mi que Pfitzner omet lorsqu'il identifie le motif initial de la pièce, ramenant sa mélodie à un «accord parfait ascendant» $(f a-l a-d o-f a)$. Berg corrige pertinemment: «Dans ce

11. Roland Barthes, Critique et Vérité, Paris, Seuil, coll. "Tel Quel», 1966. Ce rare texte ouvertement polémique de Barthes se voulait, on s'en souvient, une défense de la «nouvelle critique» contre les attaques de Picard dans son pamphlet Nouvelle critique ou nouvelle imposture (Paris, J.-J. Pauvert, coll. «Libertés», 1965), écrit notamment en réaction à l'essai du même Barthes, Sur Racine (Paris, Seuil, coll. «Pierres vives», 1963).

12. Berg, Écrits, p. 8 o. 
trait mélodique, inlassablement répété, n'est-ce pas avant tout la note mi, étrangère à l'accord de fa majeur, qui nous frappe et nous charme ${ }^{13}$ ?" [Fig. 1] Or cette apparente note de passage n'est justement pas une «note de passage » au sens classique de l'expression, c'est-à-dire une note accessoire sans identité harmonique servant à transiter mélodiquement d'un accord à un autre. Non seulement elle est étrangère à l'accord de la tonalité principale, laquelle sera d'ailleurs appelée à moduler tout au long de la pièce, mais elle crée un lien harmonique suspendu avec la tonalité de $s i$ bémol majeur, en préparant l'accord de sous-dominante sur lequel la mélodie va momentanément se reposer après son ascension légèrement retardée ${ }^{14}$. Qui plus est, c'est sur cette note étrangère, et non sur l'accord familier de fa majeur, que la mélodie s'appuie pour prendre son envol. L'effet engendré est celui d'une oscillation entre deux tonalités voisines (l'une clairement établie, l'autre simplement suggérée), soit entre deux mondes harmoniques distincts mais rapprochés, à l'image de la rêverie qu'il s'agit d'évoquer. Voilà bien un effet de style où l'esthétique fait sens musicalement. Encore fallait-il mettre le doigt sur cette note pivot fondamentale, ce que Pfitzner n'avait su faire.

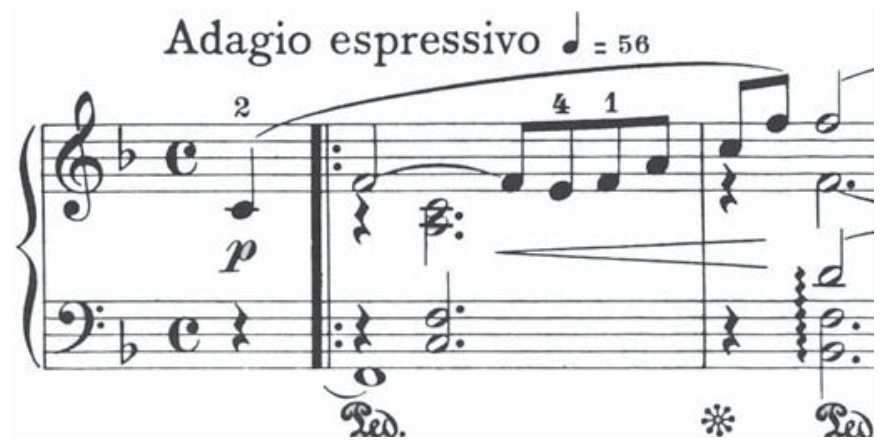

Fig. 1: Schumann, Träumerei, mesures 1 à 3 (éditions Schirmer)

Je pourrais étoffer cette analyse de Berg par bien d'autres exemples musicaux empruntés à différents compositeurs et à diverses époques. Je vais m'en tenir à deux autres échantillons tirés de la Rêverie (une autre!) de Debussy et de son illustre Clair de lune. Dans les deux cas, l'effet stylistique

13. Ibid., p. 85 .

14. Strictement parlant, l'accord mi-fa-la-do est un accord de septième qui n'appartient ni à la tonalité principale de fa majeur, dans laquelle le morceau est composé, ni à la tonalité de si bémol majeur qui constitue la sous-dominante, auquel cas le mi aurait été bémolisé. Très fugacement ici, Schumann s'affranchit des lois de la tonalité classique (voire romantique), en anticipant l'engouement pour les accords de septième et de neuvième que l'on retrouvera à satiété chez les impressionnistes français (Fauré, Debussy, Ravel) et leurs épigones. 
a un sens précis d'un point de vue musical ${ }^{15}$, mais ce sens peut échapper à qui se laisse seulement bercer par l'harmonie "impressionniste» de la musique. Le thème principal de la Rêverie de Debussy (également en $f a$ majeur) est composé de deux phrases musicales construites sur le même motif mélodique. L'harmonisation de la première phrase qui occupe huit mesures est répétitive et plutôt statique, tandis que la deuxième, également de huit mesures, reçoit un traitement harmonique changeant et très mobile. Les deux mouvements de la rêverie sont ainsi évoqués: le balancement insistant dans un ton enharmonique (sol mineur par rapport à fa majeur, le si bémol servant de note pivot) et l'écoulement fugace évoqué par l'harmonisation chatoyante de la mélodie. On passe rapidement, dans les arpèges d'accompagnement de cette deuxième phrase, d'un accord de ré mineur à des accords de la mineur, fa majeur et sol mineur; puis de l'accord de septième de dominante du ton principal (do-mi-sol-si bémol) jumelé à la neuvième (ré), aux accords de sol mineur, fa majeur, la mineur et ré mineur, avant de se reposer momentanément sur la dominante (non sans avoir effleuré au passage la sous-dominante). Tous ces accords appartenant à des tons voisins rapidement enchaînés sont traités comme des tonalités éphémères, indépendantes les unes par rapport aux autres (sauf pour la septième de dominante), mais étroitement reliées entre elles, libérées surtout des contraintes de l'harmonie classique. Or, à la fin du morceau, quand le thème est repris avant la brève coda, l'harmonisation du deuxième segment de la deuxième phrase module sans transition dans la tonalité de mi bémol majeur tandis que le ré de la mélodie, étranger à ce ton, se frotte contre le mi bémol fondamental, créant simultanément l'effet successif de suspension que Schumann avait introduit au début de sa «Rêverie» [Fig. 2]. Le sens de cette modulation n'est pas seulement d'enchanter notre oreille par un tour de passe-passe impressionniste (bien que cette magie opère réellement), mais de signaler à l'auditeur attentif que la fin approche, que le temps de la rêverie s'achève. Bref, l'effet de style agit ici comme un indice herméneutique ${ }^{16}$.

15. Contrairement à Pascal Quignard qui avance la proposition: «Il n’y a pas de point de vue sonore» (La haine de la musique, Paris, Gallimard, coll. «Folio», 1996, p. 107), je soutiens qu'une telle perspective existe en musique, et qu'elle se perçoit précisément lorsque l'on déchiffre une partition à vue. Les deux effets de style que j'analyse chez Debussy sont en effet particulièrement sensibles quand on lit la partition au piano et que l'on voit, dans une illumination soudaine, ce que l'oreille ne discerne que nébuleusement à une écoute distraite.

16. C'est le sens que donne Barthes au mot «indices» dans son article célèbre "Introduction à l'analyse structurale des récits», Communications 8, Paris, Seuil, coll. «Points», 1981: «Les indices ont donc toujours des signifiés implicites [...]. Les indices impliquent une activité de déchiffrement [...].» (p. 16-17) 


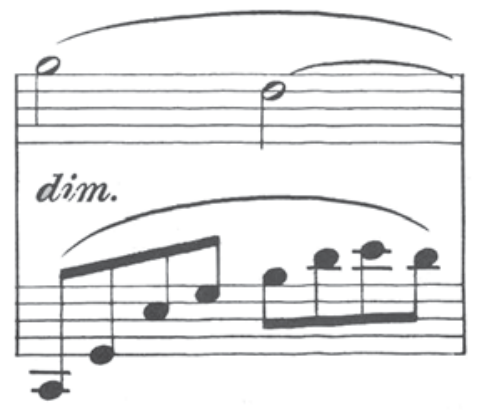

Fig. 2a: Debussy, Rêverie, mesure 15; (éditions Schirmer)

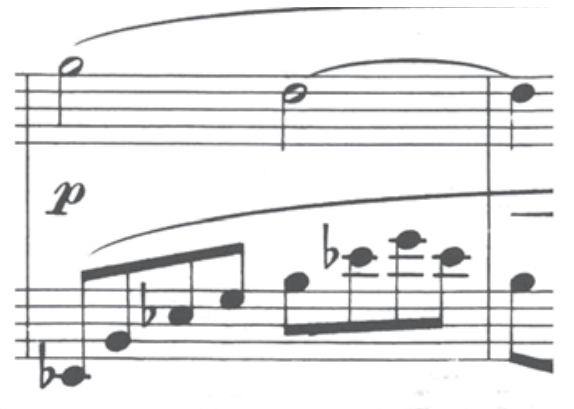

Fig. 2b: Debussy, Rêverie, mesure 88

De façon plus subtile, Debussy reprendra le procédé à la fin de son Clair de lune. La reprise à la fin de la pièce du thème principal en ré bémol majeur, qui reçoit cette fois un accompagnement plus fluide, aérien, par rapport aux accords tronqués et suspendus du début (comme si tout à coup des nuages voilaient le clair de lune statique qui se mirait dans un bassin d'eau), module très légèrement, presque imperceptiblement, quand la deuxième phrase de sa mélodie réapparaît: le ré bémol de la tonalité principale, c'est-à-dire la tonique, s'abaisse d'un ton sur la note qui deviendra le pivot inattendu de cette finale, do bémol, créant un lien enharmonique si discret entre ces deux univers sonores qu'il peut échapper à l'oreille vagabonde, laquelle subit néanmoins son pouvoir d'envoûtement [Fig. 3]. On pourrait évoquer à ce sujet un effet subliminal du style qui agit sur l'auditeur à son insu. Le mélomane pressent, plus qu'il ne le sent, qu'il s'achemine vers la fin. Il ne reste plus au compositeur qu'à conclure le morceau, la mélodie se réduisant à des échos du thème tandis que l'harmonie se volatilise dans l'air.

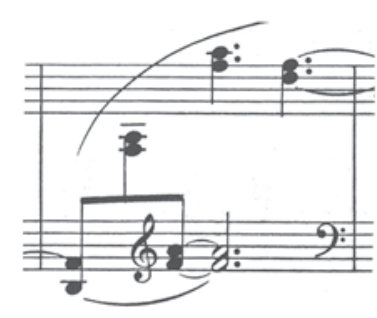

Fig. 3a: Debussy, Clair de lune, mesure 9; (éditions Schirmer)

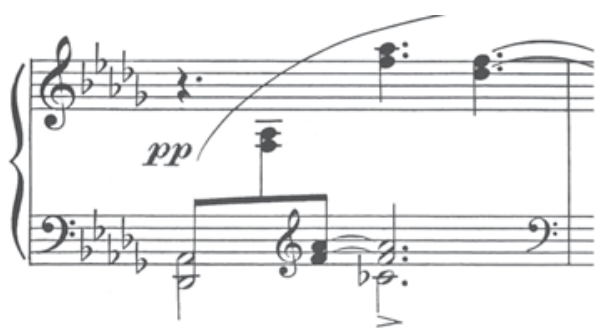

Fig. 3b: Clair de lune, mesure 59

Si nous nous transportons maintenant du côté de la littérature, ou plus généralement de l'écrit, nous trouvons également quantité d'exemples où l'effet de style ne sert pas seulement à rehausser la qualité d'un texte (ou 
même à l'hypothéquer, si nous ne goûtons pas ce style en particulier), mais concourt à l'élaboration de sa signification, soit en forçant les limites imposées par la langue à la pensée, soit en prolongeant cette dernière par le jeu des sonorités, ou encore en la soulignant par l'accentuation du rythme. Le style peut à lui seul faire entendre autrement une idée reçue, un cliché ou un stéréotype qu'il contribue ainsi à revitaliser. Je me limiterai à trois exemples ponctuels d'indices du style littéraire à l'œuvre, manœuvrant entre esthétique et herméneutique.

Nul n'a autant critiqué la notion de style que Roland Barthes, qui lui reprochait sa dimension idéologique, tributaire de l'individualisme bourgeois, son caractère ontologique qui la soustrayait au choix éthique, sa "platitude» face au "volume» de l'écriture, sa réduction rhétorique à l'opposition entre "forme» et «fond» ou linguistique à la règle de «la norme et [de] l'écart». Et pourtant, peu de critiques se sont signalés autant à ce titre au sein de la soi-disant nouvelle critique (formaliste, structuraliste et sémiotique) que cet essayiste en apparence objectif, mais en vérité plus subjectif qu'il ne le laissait paraître. On n'a qu'à relever le tissu sensuel des métaphores avec lequel il enrobe sa critique du style pour s'en convaincre. «Le style "nappe" les articulations sémantiques du contenu ${ }^{17}$ »; son "problème [...] ne peut être traité que par rapport [au] feuilleté du discours; et, pour continuer les métaphores alimentaires »:

[...] si jusqu'à présent on a $v u$ le texte sous les espèces d'un fruit à noyau (un abricot, par exemple), la pulpe étant la forme et l'amande étant le fond, il convient de le voir plutôt maintenant sous les espèces d'un oignon, agencement superposé de pelures (de niveaux, de systèmes), dont le volume ne comporte finalement aucun cour, aucun noyau, aucun secret, aucun principe irréductible, sinon l'infini même de ses enveloppes - qui n'enveloppent rien d'autre que l'ensemble même de ses surfaces ${ }^{18}$.

Le nappé et le feuilleté, qui relèvent du registre gastronomique, enrobent ou rehaussent le style et le discours d'un goût précieux - onctueux dans le premier cas, délicat dans le second -, révélateurs toutefois des goûts personnels de l'auteur (à la sauce ou à la crème épaisse du style, il préfère la légèreté du discours millefeuille). Quant au choix de l'abricot et de l'oignon pour désigner deux conceptions du texte, s'il appartient à un registre culinaire plus familier, il a été retenu en raison du jeu poétique des sonorités (l'abricot plutôt que la prune ou la pêche comme fruit à noyau) ou de la justesse de l'expression (la pulpe et non la chair, les pelures qui n'enve-

17. Roland Barthes, "Le style et son image», Essais critiques IV. Le bruissement de la langue, Paris, Seuil, 1984, p. 145.

18. Ibid., p. 150. 
loppent rien sinon l'oignon lui-même). Le style pour combattre l'idée même de style, son «image» mise à mal par une autre image jugée plus judicieuse... Il fallait Barthes, le critique, pour y penser; et Barthes, le styliste, pour l'accomplir.

Autre exemple de préciosité où la manière de l'auteur ne peut être taxée de maniérisme, ou du moins être dite maniérée au mauvais sens du $\operatorname{mot}^{19}$ : Pascal Quignard. Toute l'œuvre de cet érudit, pourtant réfractaire à la philosophie, se meut entre littérature et philosophie, en passant par la musique et la psychanalyse. Dans sa magistrale Leçon de musique, qui est moins une leçon sur la musique qu'une leçon que sa muse nous enseigne, il consacre un texte à Aristote relié aux deux autres textes de son triptyque par le truchement du thème de la «mue» (mue de la voix masculine à la puberté) qui se laisse décliner musicalement. Or, vers la fin des variations auxquelles se prête ce thème transposé dans la vie du philosophe macédonien, Quignard prépare sa coda avec une formule bien tournée; une ritournelle qui, loin de se résumer à un ornement baroque ou à une enjolivure rococo, laisse entendre le non-dit autour duquel gravitait sa réflexion sur les rapports entre mue, musique et tragédie:

Après que nous muâmes dans l'air atmosphérique, dans la pulmonation, le cri et la lumière.

Après que nous avons mué à deux fois sept ans. Après que nous avons bêlé.

Avant que nous muions dans l'absence du temps. Avant que nous muions dans d'absence du langage. Avant que nous muions dans l'absence d'espace. Avant que nous muions dans l'absence de corps ${ }^{20}$.

Aristote n'est pas encore mort, mais sa mort annoncée s'énonce par résonance dans ce court fragment musical (à la fois euphonique et eurythmique), en jouant de quelques permutations sur les temps du verbe «muer» qui se font écho. Au passé simple et au subjonctif présent, temps de la conjugaison peu fréquents à l'oral dans lesquels le verbe acquiert des sonorités rares, on entend par sympathie les harmoniques anagrammatiques de la mise à mort du bouc émissaire: muâmes/tuâmes, muions/mourions. L'écrivain musicien peut alors conclure en toute logique:

Aristote meurt. [...] Minutieusement il abandonne le jour, l'odeur, la voix, luimême. Même la voix muée, il la laisse derrière lui. La voix muée mue dans

19. Si «le maniéré est toujours un mauvais style», selon Friedrich Schleiermacher (Herméneutique, Genève, Labor et Fides, 1987, p. 148), il ne faudrait pas confondre la manière des maniéristes, qui a été tardivement reconnue comme style par les historiens de l'art, avec sa contrefaçon... maniérée. La remarque vaut tout autant pour le style "précieux» qu'il ne faudrait pas attribuer à une forme affectée de préciosité, car il y a des précieuses (et des précieux) non ridicules.

20. Pascal Quignard, La leçon de musique, Paris, Hachette, coll. «Textes du xx ${ }^{\mathrm{e}}$ siècle», 1987, p. 99. 
quelque chose de moins rauque et de moins inégal. La robe ultime qui est laissée, c'est la vie.

Un corps soudain se décompose et mute dans le silence. Il se minéralise. C'est le réel qui approche ${ }^{21}$.

La référence «muette» au réel (la mue qui mute dans la mort) me permet de transiter vers mon dernier exemple qui se veut une glose minimaliste du style réputé hermétique de Jacques Lacan. Admirée en son temps, décriée de nos jours, son œuvre psychanalytique, qui fait la part belle à l'oralité ou plutôt à l'écrit transposé à l'oral (à l'inverse de Céline qui transposait dans l'autre sens), n'a cessé de violenter la langue normative pour faire entendre l'interdit ( l'inter-dit $\left.^{22} »\right)$ d'un savoir impossible à dire (mais à lire entre les lignes). Ce qu'il m'importe de souligner dans les jeux de mots lacaniens et les formulations abstruses qui émaillent le discours de l'analyste, c'est le caractère non orthodoxe de la grammaire pour signifier l'orthodoxie de la pensée dans son rapport d'adéquation avec le réel qui ne cesse de se dérober à l'entendement. Quand Lacan joue avec la langue, la malmène, abuse d'elle, il ne se contente pas de tourner une belle formule (qui sonne d'ailleurs souvent mal à l'oreille au point de l'agresser); il la frappe au coin de la raison psychanalytique, c'est-à-dire d'une logique irrationnelle au regard de la logique rationnelle, dont la raison est à chercher ailleurs que dans la conscience (car l'inconscient a ses raisons que le conscient ne veut surtout pas entendre). Déformer avec le stylet du style le moule de la syntaxe - opération éminemment mallarméenne - était pour Lacan le meilleur moyen de faire dire à la langue ce qu'elle ne permet pas de dire, mais qu'il est néanmoins possible, voire impératif, de penser. À défaut d'enrayer le disque, il fallait le graver autrement. La stylistique lacanienne, sa fameuse "linguisterie ${ }^{23}$ », n'est rien d'autre que cette voix herméneutique qui tente de se frayer une voie heuristique dans l'écoute des signifiants et plus encore de l'insignifiant.

\section{Late Style: A Spätzeit Odyssey}

En dernière analyse, la question du style est une question de temps. Combien de temps faut-il pour mûrir un style? À moins d'être un génie précoce, formule tautologique car le "génie» est par définition précoce: pas mal de temps. Dans la dimension ontologique que Barthes lui reconnaissait, le style

21. Ibid., p. 101.

22. Jacques Lacan, Le Séminaire, livre xx. Encore, Paris, Seuil, coll. «Le champ freudien», 1975, p. 108.

23. Ibid., p. 20. 
répond à l'injonction nietzschéenne: «Devenir ce qu'on est ${ }^{24}$.» Or devenir effectivement ce que l'on est seulement en puissance, en supposant que cela se produise, met pour la plupart d'entre nous (hormis le génial ingénu) un temps considérable. Dans sa dimension "phylologique» (quelque part entre philologie et phylogénétique), c'est-à-dire en tant que produit collectif d'une époque, la question du temps n'est plus tellement celle de sa maturation que de son déclin : à partir de quel moment un style est-il jugé dépassé, périmé, désuet? Les modes «rétro» ou «néo » suffisent-elles à réactiver un certain style parvenu à échéance? L'épithète «décadent» peut-il à lui seul rescaper tel style tombé en déchéance? Ces questions intempestives mamènent in extremis à poser celle du "style tardif» (late style) et de son corollaire: le style qui retarde (belated), qui s'attarde outre mesure, qui a dépassé - sans avoir tiré sa révérence - sa date de péremption. Commence alors cette Spätzeit Odyssey, qui n'est que l'odyssée recommencée, moins de l'espace que du temps, ou mieux d'un espace-temps en défusion. Si j'ai signalé au passage divers auteurs qui ont alimenté jusqu'ici ma réflexion, je dois cette fois m'appuyer sur un essayiste en particulier que je n'attendais pas sur ce chapitre: Edward W. Said, depuis la parution de son ouvrage posthume, donc lui aussi tardif voire retardataire, On Late Style ${ }^{25}$.

La question n'est pas nouvelle, mais elle a suscité un regain d'intérêt dans le passage - pourtant si peu remarquable malgré son caractère d'épochè $\mathrm{du} \mathrm{Xx}{ }^{\mathrm{e}}$ au $\mathrm{XxI}^{\mathrm{e}}$ siècle. À différents moments de l'histoire (de l'art, de la religion, de la métaphysique, de la culture, de la civilisation...), il y a eu par intermittence, ponctuellement mais itérativement, des créateurs qui sont apparus à contre-courant de leur époque - anachroniques par rapport au temps dans lequel ils vivaient - et qui pourtant ont marqué leur siècle en produisant des œuvres qui ont passé aux yeux de la postérité pour des chefs-d'œuvre alors qu'elles avaient été perçues par le regard contemporain comme des exercices spirituels se terminant en queue de poisson, c'est-àdire en tournant le dos à l'époque qui les avait vues naître. Bach en est l'exemple par excellence dans le domaine musical à l'ère classique tardivement baroque, mais tout ce qu'on a désigné du vague vocable de postromantisme en donne des exemples plus nombreux au tournant des $\mathrm{XIX}^{e}$ et $\mathrm{xx}^{\mathrm{e}}$ siècles: Mahler, R. Strauss, Sibelius, Janácek, Busoni, Koechlin, etc. (en littérature, on pourrait leur adjoindre les noms de Th. Mann, Hofmannsthal, Strindberg, Kafka, D’Annunzio, Proust...). Pour illustrer ce cas de figure en musique, je pourrais m'appuyer sur l'exemple du jeune

24. Friedrich Nietzsche, Ecce homo, Paris, Union Générale d'Éditions, coll. «10|18», 1988, p. 55 .

25. Edward W. Said, On Late Style. Music and Literature Against the Grain, New York, Vintage Books, 2006. 
Schoenberg, mais je préfère me tourner vers deux compositeurs qui sont fort peu connus à ce titre: Theodor W. Adorno et Glenn Gould.

Said traite beaucoup d'Adorno et de Gould dans son essai posthume. Dans le premier chapitre intitulé "Timeliness and Lateness", qui tourne autour des œuvres tardives de Beethoven - «Spätstil Beethovens», selon la formule adornienne -, il insiste sur la parenté stylistique entre l'œuvre musicale des dernières années du compositeur (ses ultimes sonates et quatuors) et celle, philosophique, de son commentateur. Toutes deux lui apparaissent comme marquées par le sceau de l'éloignement, de l'exil et de l'anachronisme ${ }^{26}$. Mais ce caractère tardif et inattendu du style (sa «lateness»), qui s'oppose à sa ponctualité opportune (sa «timeliness»), n'est pas pour autant dépourvue de nouveauté («newness»), comme l'atteste Adorno à propos des innovations de la période tardive de Beethoven, annonciatrices selon lui de la révolution musicale quaccomplira un siècle plus tard Schoenberg. On pourrait même avancer que si le style tardif semble manquer de timing, c'est qu'il répond à une logique supérieure de l'atemporalité (timelessness), en tendant à s'effacer pour mieux rejoindre l'éternité. C'est ce que tout amateur de musique (au sens fort de l'expression) entendra en écoutant corps et âme les postromantiques, de Gustav Mahler au jeune Schoenberg, et sans doute quelques impressionnistes et expressionnistes de passage.

Or la musique d'Adorno, dont ne traite pas Said dans son texte, est aussi marquée par le même sceau de la Spätzeit opposée au Zeitgeist (l'esprit ou l'air du temps) qu'il abhorrait. Peu connu comme compositeur, à l'instar de Nietzsche (son «frère» en philosophie), Adorno est pourtant l'auteur d'un catalogue respectable, bien qu'il ait interdit ou différé la publication de plusieurs de ses œuvres. Proche de Schoenberg et de la tradition de la seconde école de Vienne, il était resté fidèle à la première phase atonale du maître, oscillant entre l'expressionisme et l'atonalité libre, quand ce dernier ainsi que ses disciples (Berg et Webern et, dans une moindre mesure, Krenek) se tournaient vers le dodécaphonisme érigé en système sériel. Comme le rappelle le philosophe dans ses Minima Moralia, un de ses professeurs de composition, Bernhard Sekles, croyant à tort que son élève désirait «être vraiment de [s]on temps», l'avait mis en garde contre le style

26. "There is therefore an inherent tension in late style, that abjures mere bourgeois aging and that insists on the increasing sense of apartness and exile and anachronism, which late style expresses and, more important, uses to formally sustain itself.» (Ibid., p. 17) J'ai choisi de traduire «apartness» par «éloignement», mais le mot, forgé par Said, semble recouvrir plusieurs sens: distance, séparation, mise à l'écart (dans l'espace et dans le temps), suggérant ainsi l'idée de marginalité ou de marginalisation. 
"ultramoderne» qui avait "cessé d'être moderne ${ }^{27}$ », qu'il jugeait donc comme étant déjà dépassé, ce qui ne peut paraître que paradoxal compte tenu du décalage horaire relativement peu accusé entre la période de l'avant et de l'après-guerre (une décennie à peine, deux tout au plus, séparant ces époques). Avec le temps, cependant, Adorno s'obstinera à cultiver cet idiome, tournant le dos au strict dodécaphonisme mais aussi à toutes les modes «néo» de même qu'au jazz, tout juste bons, à ses yeux, à meubler le temps libre (Freizeit), dont il disait ne pas disposer, puisqu'il prenait très au sérieux son violon d'Ingres... Il n'est pas surprenant, dès lors, qu'un autre critique ait perçu dans sa musique quelque chose de vétuste, auréolé d'un halo nostalgique ${ }^{28}$. D'une lucidité catégorique, sans compromis ni compromission, Adorno préférait nager à contre-courant du flux historique en défendant l'impératif de la "nouvelle musique». Ce faisant, il s'agissait moins pour lui de recouvrer l'aura dépérissante de l'œuvre d'art «à l'époque de sa reproduction mécanisée ${ }^{29}$ ", que de retrouver un peu de cette liberté perdue à l'ère de sa reproduction... sérielle.

Que dire alors de la production musicale d'un Glenn Gould, moins copieuse que celle du philosophe mais tout aussi «réactionnaire», sinon plus, aux yeux de la critique? Le pianiste, mort au seuil de sa deuxième carrière de chef d'orchestre et de compositeur qu'il avait à peine entamée - n'ayant produit qu'un opus officiel mais plusieurs œuvres disparates et inédites -, remonte plus loin dans le temps que le philosophe en tentant une synthèse de différentes époques de la musique occidentale. Ce fameux quatuor à cordes, opus 1, composé en 1956, soit à l'époque de l'essor de la musique dite contemporaine au Canada, plonge ses racines dans le contrepoint baroque et même prébaroque, fait le saut temporel dans les aventures formelles de l'école de Vienne, tout en restant imprégné de l'esprit postromantique germanique (teinté même d'un soupçon de romantisme honni

27. Theodor W. Adorno, Minima Moralia. Réflexion sur la vie mutilée, trad. de l'allemand par Éliane Kaufholz et Jean-René Ladmiral, Paris, Payot \& Rivages, coll. «Petite Bibliothèque Payot», 2003, p. 292.

28. "It is true that there is something antiquated about Adorno's works, compared to other pieces written at the same time, in that they hardly go beyond that which Schoenberg, Berg and Webern composed 20 years earlier. The pieces are formally in the tradition of the Second Viennese School... And yet they are written at a time when the great composers had already abandoned such forms... By evoking the spirit of the music written before the First World War, Adorno's music acquires a somewhat nostalgic quality: it expresses a longing for the great heroic period of New Music. » Dieter Schnebel, cité par Sigfried Schibli (trad. par W. Richard Rieves), dans les notes d'accompagnement du CD: Theodor W. Adorno, Kompositionen, Wergo, WER 6173-2, 1990.

29. "On pourrait réunir tous ces indices dans la notion d'aura et dire: ce qui, dans l'œuvre d'art, à l'époque de la reproduction mécanisée, dépérit, c'est son aura.» Walter Benjamin, Écrits français, Paris, Gallimard, coll. «Folio essais», 1991, p. 180-181. Dans une lettre adressée à Benjamin, Adorno abonde dans son sens, tout en lui reprochant d'avoir raté la part de liberté qui se trouve au centre de l'œuvre d'art autonome. Ibid, p. 171. 
mais aux effluves persistants). Plus qu'un simple exercice scolaire, moins qu'un chef-d'œuvre, ce quatuor de jeunesse demeure une œuvre accomplie, composée cependant à rebours de l'Histoire. Gould avait beau s'en défendre en alléguant que c'est l'opus 2 qui compte, laissant entendre par là qu'il rejoindrait bientôt ses contemporains, le fait même que ce dernier - et tous les autres opus qu'il promettait à sa suite - n'ait jamais vu le jour témoigne du contraire. En ce sens, il aura été un compositeur postmoderne bien avant la lettre, cultivant avec bonheur, parfois même avec humour, divers styles puisés à des époques historiques éloignées les unes des autres.

On Late Style retient aussi l'exemple de Thomas Mann comme un cas de style tardif en littérature, et indirectement celui de Hofmannsthal à travers les opéras de Richard Strauss dont il avait signé plusieurs livrets, opéras que Gould admirait inconditionnellement face au jugement plus mitigé d'un Adorno. Dans "Glimpses of Late Style», Said s'intéresse surtout à la longue nouvelle de Mann, Mort à Venise, et à ses adaptations lyrique (l'opéra de Britten) et cinématographique (le film de Visconti), comme figure emblématique du style littéraire tardif au $\mathrm{xx}^{\mathrm{e}}$ siècle. Mais c'est vers Le Docteur Faustus du même Mann - si l'on veut s'en tenir à une œuvre littéraire sans ses prolongements musicaux ou filmiques - qu'il faut se tourner pour apprécier pleinement le style tardif de l'auteur et prendre la juste mesure de ses accomplissements. Said aborde l'ouvre ailleurs dans son livre, mais il consacre somme toute peu de pages à cet opus magnum de Mann par rapport à son plus populaire opuscule vénitien.

On a voulu voir, derrière le personnage du compositeur Adrian Leverkühn, le portrait d'Arnold Schoenberg dont s'était inspiré Thomas Mann pour la rédaction du fameux chapitre Xxir de son roman faustien consacré au "système dodécaphonique ou sériel» de composition en régime atonal. Mais comme l'auteur reconnaissait sa propre dette envers le maître viennois dans une note ajoutée à la fin du volume, on ne fait que reconduire par là une évidence qui a maintenant valeur de truisme, qui plus est, erroné. En réalité, tout au long de ce Bildungsroman qui retrace la formation musicale (mais aussi religieuse, philosophique, existentielle, voire cosmologique) du compositeur Leverkühn - un nom qui laisse entendre le caractère audacieux, téméraire, voire héroïque, de la personnalité qu'il désigne -, telle que relatée par son ami et adulateur, Serenus Zeitblom (au nom également prédestiné), le portrait qui se dégage du personnage est multiple, variant au fil des styles qu'il assimile au cours de son développement comme musicien. Leverkühn apparaît tour à tour comme un apprenti sorcier du romantisme qui a rapidement assimilé et dépassé les leçons de son maître Kretzschmar, un impressionniste occasionnel dans ses Phosphorescences de la Mer qui rappellent la célèbre 
fantaisie symphonique de Debussy, un postromantique consommé à travers ses lieder, un expressionniste à la limite du trivial dans son Apocalypse et, in extremis, comme un démon (ou est-ce un ange?) annonciateur du dodécaphonisme atonal et sériel. Après avoir refait en accéléré l'histoire de la musique occidentale du $\mathrm{XIX}^{\mathrm{e}}$ au Xx ${ }^{\mathrm{e}}$ siècle, il s'arrête au seuil de son époque, avec sa cantate intitulée Chant de douleur du docteur Faustus qui opère un retour inattendu à Monteverdi. Mais cette cantate fait aussi signe à l'opéra inachevé que le compositeur italo-germanique Ferruccio Busoni avait composé sur le même sujet à l'époque où Mann situe la composition tardive de son personnage. Comme Leverkühn, Busoni s'était arrêté au seuil de l'atonalité, mais cela ne l'empêcha pas de prédire ce qui allait venir (entre autres, l'avènement de la musique microtonale). C'est d'ailleurs une prérogative du «late style» que d'être intensément de son temps tout en ne s'engageant pas dans la voie indiquée par lui. Dans les mots plus éloquents d'Edward Said: "Late style is what happens if art does not abdicate its rights in favor of reality ${ }^{30}$.» Ce que l'on pourrait traduire librement ainsi: «L'art a tous les droits, dont celui de ne pas adhérer à la réalité de son époque.»

La trouvaille du «late style», pour renouer in fine avec la valeur heuristique du style, n'est rien d'autre que retrouvaille. C'est le sens que prend le mot «invention» dans l'expression «l'Invention de la sainte Croix» de $L a$ Légende dorée de Jacques de Voragine, dont l'écrivain Hubert Aquin s'était inspiré pour la composition de son roman de jeunesse (paru posthume) L'Invention de la mort. L'invention comme découverte, certes, mais à condition d'entendre la syllepse de sens que recouvre le mot: création et récupération. «Inventer» signifie donc « découvrir» ou mieux «re-découvrir » ce qui était caché, celé ou oublié depuis la Création (d'où l'impression factice, mais juste, d'une création ex nihilo). Le style - et en particulier celui qui retarde sur son époque - constitue peut-être le meilleur moyen d'accéder à cette découverte herméneutique. L'artiste tardif serait cette sentinelle de l'art qui avance résolument dans le temps tout en gardant l'œil braqué sur le rétroviseur de l'histoire. Dans ce sens, il/elle est "postmoderne", avant ou après la lettre, peu importe, car d'esprit résolument intemporel, œuvrant toujours déjà dans le «pré-posthume ${ }^{31}$ » (par prescience du nihil à venir ou plutôt à revenir). Pour le dire avec les mots de Schelling paraphra-

30. Edward Said, On Late Style, p. 9.

31. C'est la traduction française approximative de l'expression allemande «Nachlass $z u$ Lebzeiten» (littéralement: "succession du vivant de l'auteur») forgée par Robert Musil pour la publication de ses « œuvres pré-posthumes». Voir l'avant-propos ironique de l'auteur dans Euvres pré-posthumes, trad. de l'allemand par Philippe Jaccottet, Paris, Seuil, 1965, p. 7-10. 
sés par Jankélévitch puis transcrits par Aquin en exergue à son dernier roman inachevé: «Le commencement n’est le commencement qu’à la fin ${ }^{32}$.»

Ergo: «Au commencement était le style, et le style était monolithique, et le monolithe était style...»

32. Hubert Aquin, «Obombre», Mélanges littéraires I. Profession: écrivain, critique établie par Claude Lamy avec la coll. de Claude Sabourin, Montréal, Bibliothèque québécoise, 1995, p. 337. La citation attribuée à Schelling se trouve dans l'ouvrage de Vladimir Jankélévitch: L'irréversible et la nostalgie, Paris, Flammarion, coll. "Nouvelle bibliothèque scientifique", 1974, p. 20. Jankélévitch condense en une formule lapidaire quelques passages tirés de Philosophie de la mythologie (trad. par Alain Pernet, Grenoble, Jérôme Millon, 1994, p. 186 et p. 428) et Philosophie de la révélation (trad. par la RCP Schellingiana, Paris, PUF, coll. "Épiméthée», 1989, Livre II, p. 344 et Livre III, p. 268) du philosophe allemand F. W. J. Schelling. 